\title{
EVALUATION OF FRACTURE RESISTANCE AND COLOR STABILITY OF INNOVATIVE ESTHETIC CROWNS FOR PRIMARY POSTERIOR MOLARS
}

\author{
Sherine Badr Youness Badr *, Mohammad Rayyan** and Manal Ahmed Elshiekh***
}

\begin{abstract}
Purpose: To evaluate color stability and fracture resistance of CAD/CAM and manually fabricated tooth colored materials as crowns on primary molars.

Methodology : Eighty epoxy replicas (Vertex- Dental B.V., The Netherlands) of minimally prepared primary molar were constructed to receive a tooth colored crown. Replicas were assigned into 4 groups $(\mathrm{n}=20)$ according to crown material used: CAD/CAM PMMA blocks (Telio Cad, Ivoclar Vivadent AG, Liechtenstein) [CC], self-polymerizing temporary resin material [SP] (Snap, Parkell, NY 11717 USA), auto-mix temporary resin material [AT] (Telio CS C\&B Ivoclar Vivadent AG, Liechtenstein), and thermoplastic resin material [TP] (DurAcetal, Myerson LLC, Chicago, IL, USA). After cementation using glass ionomer cement (Vivaglass CEM, Ivoclar Vivadent AG, Liechtenstein), all specimens were thermo-cycled for 1000 cycles. Tooth shade was recorded using spectrophotometer, after storing in: orange juice, and carbonated beverage. Fracture resistance was assessed by axially loading the crowns using a universal testing machine . Data were analyzed using one-way analysis of variance and post hoc tests $(\alpha=0.05)$.
\end{abstract}

Results: Spectrophotometric analysis revealed unacceptable color change of manually fabricated crowns; $\mathrm{SP}(\Delta \mathrm{E}=5.4 \pm 1.6)$, $\mathrm{AM}(\Delta \mathrm{E}=6.7 \pm 1.2)$ and $\mathrm{TP}(\Delta \mathrm{E}=4.4 \pm 2.5)$. On the other hand, $\mathrm{CC}$ crowns demonstrated high color stability $(\Delta \mathrm{E}=1.7 \pm 0.2)$. CAD/CAM crowns demonstrated significantly higher fracture resistance $(1417.9 \pm 62 \mathrm{~N})$ compared to manually fabricated crowns $(\mathrm{SP}=1096 \pm 50, \mathrm{AM}=989 \pm 41$ and $\mathrm{TP}=1297 \pm 45)$.

Conclusions: CAD/CAM PMMA crowns revealed superior fracture resistance and color stability properties suitable for long-term clinical performance within the primary molars.

KEYWORDS: CAD CAM, crown, PMMA, thermoplastic, fracture resistance and color stability.

\footnotetext{
* Professor, Professor of Pediatric Dentistry and Dental Public Health, Faculty of Dentistry Cairo University. ** Professor, Professor and Chairperson of Fixed Prosthodontics Department Sinai University, Kantara Campus.

*** Associate Professor, Associate Professor of Pediatric Dentistry and Dental Public Health, Faculty of Dentistry Cairo University.
} 


\section{INTRODUCTION}

Stainless steel crowns served as the most commonly used full coverage option for badly decayed or pulpally treated primary molars. They were definitely considered as the golden standard of pediatric crowns. This is because of their numerous advantages as being affordable and adjustable in addition to their reliability and durability. ${ }^{(1)}$

Unfortunately, their metallic nature poses two main draw-backs: unaesthetic color and bio-incompatibility. This unpleasant esthetic negatively influences both child and parent's psychology and can't be overlooked or neglected. An essential part of any pediatric dentist armamentarium nowadays is to offer an esthetic full coverage posterior restoration to his patients whenever needed. This is to guarantee offering the best treatment option for better patient satisfaction and better quality of life. ${ }^{(2,3)}$

The continuous increased esthetic demand of both parents and children, led to the introduction of several esthetic options. However, all available esthetic alternatives have limitations; open faced crowns require more chairside time, esthetically unsatisfactory. Pre-veneered crowns are bulky, lack natural appearance and have limited crimping ability negatively affect their durability and overall clinical performance. Moreover, they are expensive and need lengthily sessions as the teeth have to be over prepared to fit the crowns with the need of fitting trials, particularly for unexperienced practitioners. $(4,5)$ More recently, the introduction of zirconia crowns offered not only a high esthetic alternative but also eliminated the problem of metallic allergy. (6) Unfortunately they have also some limitations as being ready-made with specific sizes completely lacking any ability to be adjusted or contoured, they also require over reduction of the tooth structure, their retention relay purely on the cement, moreover they require extra training, knowledge and chair time. Above all, they are considered an expensive esthetic alternative. ${ }^{(7,8)}$
The urge for an esthetic option for primary dentition have urged both researchers and dental companies to find a reliable solution for this dilemma either in materials or techniques. Taking into consideration; durability, retentiveness, adaptability, placement time, reparability and last but not the least cost effectiveness.

Long-term temporization concept was introduced in adult permanent dentition as it was needed in special situations including implant dentistry and comprehensive occlusal adjustment. ${ }^{(9)}$ These materials are also used in thin sections as in the case of veneer temporization under adult occlusal loads. ${ }^{(10)}$ The used material should have optimal mechanical qualities, marginal integrity, and color stability to guarantee its performance as long-term adult provisional restoration. ${ }^{(11)}$

Different materials are currently available for fabrication of adult provisionals starting from conventional acrylics based on methyl methacrylate resins which offer a cheap handy material. On the other hand, they stain easily, lacks marginal integrity, and suffer from residual monomers leaching which intern leads to soft tissue irritation. ${ }^{(12)}$ Bis-Acryl and Bis-GMA based materials are superior in their color stability, mechanical properties and have less polymerization shrinkage compared to acrylic resin based materials. ${ }^{(13,14)}$

Computer assisted design/computer assisted machining (CAD/CAM) adult provisionals are milled from acrylic blocks which are fabricated under controlled and precise conditions. These temporaries are more color stable with better marginal quality compared to conventional ones. ${ }^{(15,16)}$ In addition to their ease of manufacturing, they acquire high polish with gentle brushing-using soft brush which prevents irritation to marginal gingiva ${ }^{(17)}$

Recently thermoplastic materials were introduced into the market. They were claimed by the manufacturer to offer superior physical and mechanical qualities for long-term provisional and for definitive removable partial dentures. 
Based on what was mentioned, the authors suggested that long-term adult temporary crowns may offer a rich pool for esthetic alternatives for primary dentition. Current study was designed to test in-vitro the color change and fracture resistance of four long term adult provisional materials; $\mathrm{CAD} /$ CAM PMMA (CC), self-polymerizing temporary resin material (SP), auto-mix temporary resin material (AM), and thermoplastic resin material (TP), in an attempt to validate their use intraorally on primary molars. The null hypothesis of current trial suggested that, there will be no significant difference between tested groups.

\section{MATERIALS AND METHODS}

A second primary maxillary molar was reduced $1 \mathrm{~mm}$ occlusaly with $0.4 \mathrm{~mm}$ chamfer finish line, to accept full coverage crown. Tooth was then inserted in a base of epoxy resin. A silicon impression (Virtual, Ivoclar Vivadent AG, Liechtenstein) was made and poured to build 80 epoxy and 10 stone dies. (Fig. 1)

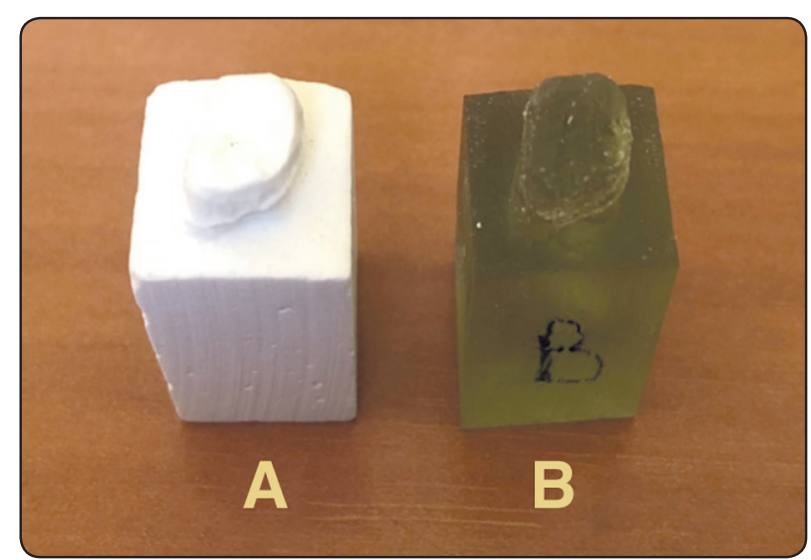

Fig. (1) A: Stone die for TP group only. B: Resin die for all other groups.

\section{Fabrication of the crowns}

For CC group: ACAD model for a full anatomical primary molar was produced after die scanning. Using CAD CAM, 20 crowns were milled from a Poly-Methyl-Methacrylate (PMMA) blank (Telio
CAD, Ivoclar Vivadent AG, Liechtenstein). Silicon impressions (Hydrorise, Zhermack, BadiaPolesine, Italy) were made for the milled CAD/CAM crown. These impressions were utilized as templates for SP, $\mathrm{AM}$ and TP groups.

For SP group: A separating medium (Vaseline) was painted on resin dies to avoid adherence of provisional material. Ethyl-Methacrylate Acrylic resin (Snap, Parkell, NY, USA) liquid and powder were manipulated according to manufacturer directions and placed into the silicon template which was inverted onto each die until complete setting. Twenty crowns were fabricated and then polished using different grit points (Acrypoint, Shofu, Kyoto 605-0983, Japan).

For AM Group: Two component auto-mixing cartridge (Telio CS C\&B Ivoclar Vivadent AG, Liechtenstein) was mounted into a dispensing syringe to be injected into the silicon template and replaced on each resin die. As formerly described, finishing and polishing were done to the 20 crowns after complete setting.

For TP Group: Silicon template was divided into 2 parts with access-hole bored on each part. Thermoplastic pellets (Duracetal, Myerson LLC, USA) were placed into a specific heating gun to be injected into the two template halves after being reassembled on preheated stone die. (Table 1) presents specimens grouping and (table 2) presents the details of the used materials.

TABLE (1) Specimens grouping.

\begin{tabular}{|c|c|c|}
\hline Group & Crown Material & Number \\
\hline CC & CAD/CAM PMMA block & 20 \\
\hline SP & Self-polymerizing MMA/PMMA & 20 \\
\hline AM & $\begin{array}{c}\text { Auto-mix Bis-acrylic self-curing } \\
\text { compound }\end{array}$ & 20 \\
\hline TP & $\begin{array}{c}\text { Thermoplastic Highly crystalline } \\
\text { acetal copolymer resin }\end{array}$ & 20 \\
\hline Total & & 80 \\
\hline
\end{tabular}


TABLE (2) Materials used.

\begin{tabular}{|c|c|c|c|c|}
\hline Material & Trade name & Composition & Shade & Manufacturer \\
\hline $\begin{array}{c}\text { Self-polymerizing } \\
(\mathrm{SP})\end{array}$ & Snap & MMA/PMMA & A2 & $\begin{array}{c}\text { Parkell, 300 Executive Drive, } \\
\text { Edgewood, NY 11717 USA }\end{array}$ \\
\hline $\begin{array}{c}\text { Auto-mix } \\
(\mathrm{AM})\end{array}$ & Telio CS C\&B & $\begin{array}{c}\text { Bis-acrylic selfcuring } \\
\text { compound }\end{array}$ & A2 & Ivoclar Vivadent AG, Liechtenstein \\
\hline $\begin{array}{c}\text { Thermoplastic resin } \\
(\mathrm{TP})\end{array}$ & DurAcetal & $\begin{array}{c}\text { Acetal copolymer resin } \\
\text { that is highly crystalline }\end{array}$ & A2 & $\begin{array}{c}\text { Myerson LLC, Ravenswood, } \\
\text { Chicago, IL, USA }\end{array}$ \\
\hline $\begin{array}{c}\text { CAD/CAM PMMA } \\
\text { block (CC) }\end{array}$ & $\begin{array}{c}\text { Telio CAD Polymethyl } \\
\text { methacrylate blocks }\end{array}$ & $\begin{array}{c}\text { CAD/ CAM Polymethyl } \\
\text { methacrylate }\end{array}$ & A2 & Ivoclar Vivadent AG, Liechtenstein \\
\hline
\end{tabular}

\section{Cementation of the crowns:}

Glass-ionomer cement (Vivaglass CEM, Ivoclar Vivadent AG, Liechtenstein) was used for cementation after mixing it on a glass slab using a special spatula (Novatech spatula, Hu-Friedy, USA). The cement was used to fill up every crown which was then placed on its corresponding epoxy die under a $5 \mathrm{Kg}$ load. After $30 \mathrm{~min}$., excess cement was removed. All specimens were stored for 72 hours prior to testing in distilled water at roomtemperature.

\section{Color stability test:}

Color changes of crowns were assessed according to Commission Internationale de l'Eclairage (CIE) $\mathrm{L}^{*}, \mathrm{a}^{*}, \mathrm{~b}^{*}$ (LAB) color scale. This scale quantitatively determines color by using 3 coordinates; $\mathrm{L}^{*}, \mathrm{a}^{*}$ and $\mathrm{b}^{*}$. On that scale; $\mathrm{L}^{*}$ is to assess an object brightness quantitatively on a scale, where black has a (0) $\mathrm{L}^{*}$ value and totally reflected light has a (100) $\mathrm{L}^{*}$ value. On the scale, $\mathrm{a}^{*}$ measures the amount of red $\left(+\mathrm{a}^{*}\right)$ and green $\left(-\mathrm{a}^{*}\right)$, where $b^{*}$ measures the amount of yellow $\left(+b^{*}\right)$ and blue $\left(-b^{*}\right){ }^{(18)}$

$\mathrm{L}^{*}, \mathrm{a}^{*}$ and $\mathrm{b}^{*}$ color coordinates (CIE Lab) were assessed using dental spectrophotometer (Easy shade Advance, Vita, Cal) directly after cementation of crowns. To avoid external light reflection, a dark box was used. All specimens were stored for a week in orange juice followed by another week in carbonated coca cola (Pepsi). Each solution was refreshed every 12 hours and surfaces of each crown were hand-brushed using a tooth paste and a soft tooth brush every 12 hours. (Fig. 2) Color coordinates were assessed again after 2 weeks and color variation was calculated using this equation:

$$
\Delta \mathrm{E}=\left[(\mathrm{L}-\mathrm{L} 1)^{2}+(\mathrm{a}-\mathrm{a} 1)^{2}+(\mathrm{b}-\mathrm{b} 1)^{2}\right] 0.5
$$

Where $\Delta \mathrm{E}$ is the color change, while $\mathrm{L}, \mathrm{a}$ and $\mathrm{b}$ are the color coordinates after cementation and L1, a1 and b1 are color parameters after storing in the staining solutions. $\Delta \mathrm{E}>3.3$ was determined as unacceptable change of color perceived by naked eye. ${ }^{(19)}$

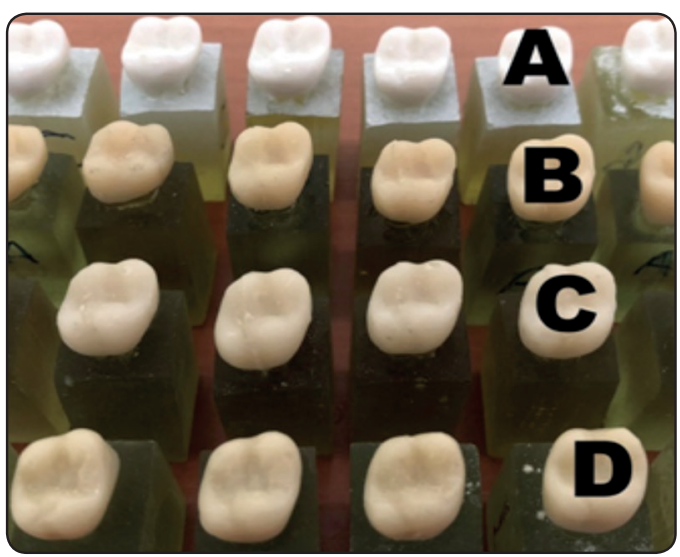

Fig. (2) stability test. A: CC group, B: AM group, C: SP group, D: TP group.

\section{Fracture resistance:}

Thermo-cycling program of 1000 cycles with temperature between 5 and $55^{\circ} \mathrm{C}$ and dwell time of 
$30 \mathrm{sec}$ was done to the cemented crowns. ${ }^{(20)} \mathrm{By}$ the use of a universal testing machine (Instron 8874; Instron Corp. Canton, Mass), the fracture resistance was determined by axial load application on cemented crowns. Loading was performed at a $1 \mathrm{~mm} /$ min cross-head speed until failure occurred. ${ }^{(21)}$

\section{Statistical analysis:}

Data were analyzed using SPSS 18.0 computer software (SPSS, Inc, Chicago, USA) where, oneway analysis of variance (ANOVA) and Bonferroni post hoc test were used at $(\alpha=0.05)$. According to the tests of choice a sample size ( $n=20 /$ group), had adequate power to detect clinically relevant variances.

\section{RESULTS}

Data analysis using spectrophotometer revealed unacceptable color change of manually fabricated specimens; $\mathrm{SP}(\Delta \mathrm{E}=5.4 \pm 1.6), \mathrm{AM}(\Delta \mathrm{E}=6.7 \pm 1.2)$ and $\mathrm{TP}(\Delta \mathrm{E}=4.4 \pm 2.5)$ while $\mathrm{CC}$ specimens maintained their original color $(\Delta \mathrm{E}=1.7 \pm 0.2)$. Crowns become darker in color with a significant decrease observed in the value of L in all manually fabricated specimens. Concerning fracture resistance, $\mathrm{CAD} /$ CAM crowns showed also a statistically significant higher mean at $(\mathrm{P}<0.05)$ compared to manually fabricated crowns. Where the mean $( \pm \mathrm{SD})$ for $\mathrm{CC}$ group was $(1417.9 \pm 62)$ and for manually fabricated crowns were SP $(1096 \pm 50)$, AM $(989 \pm 41)$ and TP $(1297 \pm 45)$ (Table 3).

\section{DISCUSSION}

The need for esthetic alternative full coverage restoration for primary molars is a highly perused objective for pediatric dentists. Thus searching for a new material becomes an urgent demand in an attempt to find a cost effective, reparable and custommade material. Such material might allow conservative preparations, better fitting, better retention, shorter chairside time and much easier placement protocol. Thus, in this research the 4 tested materials were selected based on their ability to offer a solution that fulfill most of those parameters

Although the use of natural teeth in in-vitro studies simulate to a great extend the clinical situation, yet in this study resin dies were used. This was done in order to eliminate the wide variability in natural teeth, as each extracted tooth has its own history and its own fracture resistance dictated by its anatomy and hard tissue thickness. Moreover, modules of elasticity of resin dies closely resembles that of natural teeth. ${ }^{(22)}$

All selected materials used in this study have their modulus of elasticity close to that of enamel. Thus, they offer some degree of stress-breaking and cushioning effect to PDL and supporting structures of both the restored tooth and opposite dentition. Moreover, all of those materials offer the advantage of being easily repaired intraorally. They are customly fabricated thus, they are better adapted to the prepared tooth than the prefabricated alternatives. They solve the problem of missing

TABLE (3) Testing results of fracture resistance among different groups.

\begin{tabular}{|l|l|l|l|}
\hline Group & Material & Mean $( \pm \mathrm{SD}) \Delta \mathrm{E}$ & Mean $( \pm \mathrm{SD})$ Fracture resistance \\
\hline CC & CAD/CAM Cercon & $1.7 \pm 0.2 *$ & $1417.9 \pm 62^{*}$ \\
\hline SP & Alike & $5.4 \pm 1.6$ & $1096 \pm 50$ \\
\hline AM & Acrytemp & $6.7 \pm 1.2$ & $989 \pm 41$ \\
\hline TP & DurAcetal & $4.4 \pm 2.5$ & $1297 \pm 45$ \\
\hline
\end{tabular}

* Statistically significant at $P<0.05$ 
sizes and unfitting sizes in prefabricated kits which often leads to over reduction and more chairside time. Also they offer better fitness and better retention. Self-polymerizing PMMA and Auto-mix Bis-acrylic materials don't need any special tools or pliers and can be easily constructed and are highly cost effective. In conjunction with intraoral scanner, CAD CAM crowns can be effectively fabricated chair-side in the clinic.

The results of this study showed significantly higher color stability of CAD-CAM PMMA material over the manually-fabricated materials that demonstrated unacceptable color change, however this change might be convenient within the context of primary molars. This color stability results were in agreement to those reported by Elagra, et al, in 2017 who concluded that CAD-CAM PMMA material exhibited superior color stability compared to Bis-acryl resin composite material, which demonstrated clinically noticeable color change. ${ }^{(23)}$ Similarly, Stawarczyk, et al, in 2012 studied color stability of manually fabricated acrylic resins and industrially fabricated CAD/CAM blocks against glass ceramic materials. They found that the discoloration of the manuallyfabricated acrylic was significantly higher compared to industrially-fabricated CAD/CAM and glass-ceramics. ${ }^{(24)}$ Also Jalil et al, in 2012 investigated the color stability of provisional restorative materials and concluded that methyl methacrylate base resin materials were found to be more color stable than butyl methacrylate in the tea solution. (25) Turgut et al, compared discoloration of provisional restorations after oral rinses and reported that; PMMA-based materials displayed less discoloration than Bis-acrylbased provisional materials. ${ }^{(26)}$

Concerning the results of fracture resistance, the lower fracture resistance values of manually fabricated crowns observed in this study can be justified based on the method of polymerization and the higher residual monomer ratio compared to $\mathrm{CAD} / \mathrm{CAM}$ crowns. ${ }^{(27,28)}$ However, all tested specimens exceeded the $600 \mathrm{~N}$ which exceeds the expected load in the posterior region in children starting from early primary to permanent dentition stage. As the mean occlusal biting forces were found by Owais AI et al., in 2013 to be $176 \mathrm{~N}$ in the early primary dentition, $240 \mathrm{~N}$ in late primary dentition, $289 \mathrm{~N}$ in the early mixed dentition, $433 \mathrm{~N}$ in the late mixed dentition. ${ }^{(29)}$

The observed fracture resistance results are consistent with results reported by Yao, et al, in 2014 who found that CAD/CAM provisional materials displayed superior strength than bisacryl provisional materials, especially after thermal cycling. ${ }^{(30)}$ But current obtained results were almost double that reported by Wanner ${ }^{(31)}$ who compared the mechanical properties of 3 different types PMMA blocks. This may be due to different PMMA CAD CAM blocks used. On the other hand, Abdullah, et al in 2016 reported that not all CAD/CAM provisional crowns displayed superior fracture strength to that of the direct provisional material, which may be resulted from different methodology adopted. ${ }^{(32)}$

CAD/CAM PMMA blocks are industrially manufactured under optimum conditions, offering superior physical and mechanical properties to manually-fabricated. ${ }^{(33-35)}$ Their good mechanical properties and color stability represent an ideal esthetic alternative for primary molars that have relatively short life time and are exposed to lesser occlusal forces compared to permanent teeth. The null hypothesis was rejected as there were significant difference among tested groups.

\section{CONCLUSIONS}

CAD/CAM PMMA crowns revealed superior color stability and fracture resistance over manually fabricated tooth colored materials. Thus these crowns may present a good esthetic substitute for primary molars crowns with expected good clinical performance that needs to be furtherly investigated in-vivo. 


\section{RECOMMENDATION}

Further in-vivo studies are needed to confirm CAD/CAM PMMA crowns performance intraorally.

\section{CLINICAL IMPLICATIONS}

$\mathrm{CAD} / \mathrm{CAM}$ and manually fabricated tooth colored materials are suitable for use in pediatric dentistry. They could provide the required fracture resistance and convenient color stability to serve as esthetic full coverage restoration for compromised primary molars.

\section{REFERENCES}

1. Seale NS. The use of stainless steel crowns. Pediatr Dent. 2002 Sep-Oct;24(5):501-5.

2. Holsinger DM, Wells MH, Scarbecz M, Donaldson M. Clinical evaluation and parental satisfaction with pediatric zirconia anterior crowns. Pediatr Dent. 2016;38(3):192-7.

3. Pani SC, Saffan AA, AlHobail S, Bin Salem F, AlFuraih A, AlTamimi M. Esthetic concerns and acceptability of treatment modalities in primary teeth: A comparison between children and their parents. Int J Dent. 2016;2016:3163904.

4. Elqadir AJ, Shapira J, Ziskind K, Ram D. [Esthetic restorations of primary anterior teeth]. Refuat Hapeh Vehashinayi. 2013 Apr; 30(2):54-60, 82.

5. Gaurav Kumar Mittal, Aviral Verma1, Hansika Pahuja, Shashank Agarwal, Himani Tomar. Esthetic Crowns in Pediatric Dentistry: A_review. International Journal of Contemporary Medical Research, 2016 May 2016; 33 (5):1280-82.

6. Kulkarni P, Agrawal S, Bansal A, Jain A, Tiwari U, Anand A. Assessment of nickel release from various dental appliances used routinely in pediatric dentistry. Indian $\mathrm{J}$ Dent. 2016 Apr-Jun;7(2):81-5.

7. Ashima G, Sarabjot KB, Gauba K, Mittal HC. Zirconia crowns for rehabilitation of decayed primary incisors: an esthetic alternative. J Clin Pediatr Dent. 2014 Fall; 39(1):18-22.

8. Lee JH. Guided tooth preparation for a pediatric zirconia crown. J Am Dent Assoc. 2018 Mar;149(3):202-208.

9. Lodding DW. Long-term esthetic provisional restorations in dentistry. Curr Opin Cosmet Dent. 1997;4:16-21.
10. Hochman N, Zalkind M. Laminate veneer provisionalisation. Eur J Prosthodont Restor Dent. 1997 Mar;5(1):31-4.

11. Trushkowsky RD. Fabrication of a fixed provisional restoration utilizing a light-curing acrylic resin. Quintessence Int. 1992 Jun;23(6):415-9.

12. Wassell RW, St George G, Ingledew RP, Steele JG. Crowns and other extra-coronal restorations: provisional restorations. Br Dent J. 2002 Jun 15;192(11):619-22, 625-30.

13. Gegauff AG, Holloway JA. Interim fixed restorations. In: Contemporary Fixed Prosthodontics. Rosensteil SF, Land MF, Fujimoto J, eds. 4th edition. St. Louis, MO: Mosby Elsevier; 20016:466-504.

14. Strassler HE, Anolik C, Frey C. High-strength, aesthetic provisional restorations using a bis-acryl composite. Dent Today. 2007;26(11): 128, 130-133.

15. Alt V, Hannig M, Wöstmann B, Balkenhol M. Fracture strength of temporary fixed partial dentures: CAD/CAM versus directly fabricated restorations. Dent Mater. 2011 Apr;27(4):339-47.

16. Stawarczyk B, Ender A, Trottmann A, Özcan M, Fischer J, Hämmerle $\mathrm{CH}$. Load-bearing capacity of CAD/CAM milled polymeric three-unit fixed dental prostheses: effect of aging regimens. Clin Oral Investig. 2012 Dec;16(6):1669-77.

17. Salimi H, Savabi O, Nejatidanesh F. Current results and trends in platform switching. Dent Res J. 2011 Dec;8(Suppl 1): S30-6.

18. Oliveira, C. B. d., Maia, L. G. M., Santos-Pinto, A., \& Gandini Júnior, L. G. In vitro study of color stability of polycrystalline and monocrystalline ceramic brackets. Dental press journal of orthodontics.2014; 19(4), 114-121.

19. Douglas, R. D., \& Brewer, J. D. Acceptability of shade differences in metal ceramic crowns. The Journal of prosthetic dentistry, 1998; 79(3), 254-260.

20. Gresnigt, M. M., Özcan, M., van den Houten, M. L., Schipper, L., \& Cune, M. S. Fracture strength, failure type and Weibull characteristics of lithium disilicate and multiphase resin composite endocrowns under axial and lateral forces. Dental Materials.2016; 32(5), 607-614.

21. Lehmann F, Eickemeyer G, Rammelsberg P. Fracture resistance of metal-free composite crowns-effects of fiber reinforcement, thermal cycling, and cementation technique. J Prosthet Dent 2004;92:258-264. 
22. Cho L, Song H, Koak J, Heo S. Marginal accuracy and fracture strength of ceromer/fiber-reinforced composite crowns: effect of variations in preparation design. J Prosthet Dent.2002 Oct; 88(4):388-95.

23. Elagra MI, Rayyan MR, Alhomaidhi MM, Alanaziy AA, Alnefaie MO. Color stability and marginal integrity of interim crowns: An in vitro study. Eur J Dent. 2017 JulSep;11(3):330-334

24. Stawarczyk B, Sener B, Trottmann A, Roos M, Ozcan M, Hämmerle $\mathrm{CH}$. Discoloration of manually fabricated resins and industrially fabricated CAD/CAM blocks versus glassceramic: effect of storage media, duration, and subsequent polishing. Dent Mater J. 2012;31(3):377-83.

25. Jalali H, Dorriz H, Hoseinkhezri F, Emadian Razavi SF. In vitro color stability of provisional restorative materials. Indian J Dent Res. 2012 May-Jun;23(3):388-92.

26. Turgut S, Bagis B, Ayaz EA, Ulusoy KU, Altintas SH, Korkmaz FM, Bagis N. Discoloration of provisional restorations after oral rinses. Int J Med Sci. 2013 Aug 30;10(11):1503-9.

27. Meloto CB, Silva-Concilio LR, Machado C, Ribeiro MC, Joia FA, Rizzatti-Barbosa CM. Water sorption of heat-polymerized acrylic resins processed in mono and bimaxillary flasks. Braz Dent J 2006;17:122-125.

28. Scherrer SS, Wiskott AH, Coto-Hunziker V, Belser UC Monotonic flexure and fatigue strength of composites for provisional and definitive restorations. J Prosthet Dent 2003;89:579-588.
29. Owais AI, Shaweesh M, Abu Alhaija ES. Maximum occusal bite force for children in different dentition stages. Eur J Orthod. 2013 Aug;35(4):427-33.

30. Yao J, Li J, Wang Y, Huang H. Comparison of the flexural strength and marginal accuracy of traditional and CAD/ CAM interim materials before and after thermal cycling. J Prosthet Dent. 2014 Sep;112(3):649-57.

31. Telio CAD. Available from: http://www.ivoclarvivadent. com/en/all/ products/resin-based-veneering-cadcammaterials/cad-cam-acrylic- polymer-blocks/telio-cad [Last accessed on 2018 July 23].

32. Abdullah AO, Tsitrou EA, Pollington S. Comparative in vitro evaluation of CAD/CAM vs conventional provisional crowns. J Appl Oral Sci. 2016 May-Jun;24(3):258-63.

33. Alt V, Hannig M, Wöstmann B, Balkenhol M. Fracture strength of temporary fixed partial dentures: CAD/CAM versus directly fabricated restorations. Dent Mater. 2011 Apr;27(4):339-47.

34. Göncü Başaran E, Ayna E, Vallittu PK, Lassila LV. Loadbearing capacity of handmade and computer-aided design-computer-aided manufacturing-fabricated three-unit fixed dental prostheses of particulate filler composite. Acta Odontol Scand. 2011 May;69(3):144-50.

35. Stawarczyk B, Ender A, Trottmann A, Özcan M, Fischer $\mathrm{J}$, Hämmerle $\mathrm{CH}$. Load-bearing capacity of CAD/CAM milled polymeric three-unit fixed dental prostheses: effect of aging regimens. Clin Oral Investig. 2012 Dec;16(6):1669-77. 